\title{
Asking for help: Do dogs take into account prior experiences with people?
}

\author{
Fabricio Carballo ${ }^{1}$ Camila Cavalli ${ }^{2,3} \cdot$ Magalí Martínez $^{2,3} \cdot$ Victoria Dzik $^{2,3} \cdot$ Mariana Bentosela $^{2,3,4}$ \\ Published online: 20 April 2020 \\ (C) The Psychonomic Society, Inc. 2020
}

\begin{abstract}
When confronted with a difficult or impossible problem, dogs tend to look back at humans and try to catch their attention, instead of trying to solve it themselves. This behavior has been interpreted as a help request, but it is debated whether dogs take into account prior experiences with people when selecting whom to turn to. In the present study, dogs were trained to discriminate between a generous experimenter who gave them food and a selfish one that took it away. After assessing that they had established a preference for the generous one, we exposed them to an unsolvable task in which food was locked inside a container, while the experimenters stood on each side of the apparatus. During this task, we measured their behaviors towards each experimenter. Results showed that dogs did not first turn to the generous experimenter. However, they gazed more at the generous experimenter during the task, which implies that they did, to some degree, selectively ask for help based on previous interactions. Moreover, they gazed more and spent significantly more time in contact with the female experimenter when she was generous, suggesting a possible synergic effect of the experimenters' ID (male/female) and their attitude (generous/selfish). All in all, these results suggest that, to some extent, dogs are able to use the information from previous interactions with unknown humans to selectively ask for help.
\end{abstract}

Keywords Human reputation $\cdot$ Unsolvable task $\cdot$ Asking for help $\cdot$ Dogs

Dogs are particularly skilled at communicating with people, and gazing at the human face plays a fundamental role in this ability (e.g., Prato-Previde \& Marshall-Pescini, 2014). One pioneer study that focused on gazing as a communicative signal is that of Miklósi et al. (2003), in which dogs were exposed to two kind of problems: pulling a rope with food attached to

Mariana Bentosela

marianabentosela@gmail.com

1 Instituto de investigaciones Biológicas y Biomédicas del Sur (INBIOSUR; CONICET-UNS), Grupo de Investigación del Comportamiento en Cánidos, San Juan 670 Piso 1 (8000), Bahía Blanca, Argentina

2 Facultad de Medicina, Instituto de investigaciones Médicas A. Lanari, Universidad de Buenos Aires, Combatientes de Malvinas 3150, 1427 Buenos Aires, Argentina

3 Consejo Nacional de Investigaciones Científicas y Técnicas, Instituto de investigaciones Médicas (IDIM), Grupo de Investigación del Comportamiento en Cánidos (ICOC), Universidad de Buenos Aires, Buenos Aires, Argentina

4 Centro de Altos Estudios en Ciencias Humanas y de la Salud (CAECIHS-UAI), Universidad Abierta Interamericana, Buenos Aires, Argentina one end and opening a container of food. After several trials in which they could successfully solve the task, they were exposed to an unsolvable trial in which the rope and the container were blocked, making food inaccessible. Results showed that dogs tend to gaze to the human face when they are faced with an unsolvable problem, which has been interpreted as a way of asking for help. Moreover, dogs are also able to use gaze alternation to direct the attention of an observer towards a target object (the unsolvable problem in this case), by looking at the partner and consecutively looking to the target or vice versa (e.g., Miklósi, Polgárdi, Topál, \& Csányi, 2000). This behavior is related to referential signaling, as it involves directing the attention of an observer to a distant object (Leavens, Hopkins, \& Thomas, 2004).

From then on, several studies have focused on dogs' reactions to unsolvable or difficult tasks. Their findings confirm that, in these situations, dogs tend to gaze at people instead of trying to solve the problem by themselves (e.g., MarshallPescini, Passalacqua, Barnard, Valsecchi, \& Prato-Previde, 2009; Udell, 2015). Furthermore, there is a negative correlation between gazing behavior and persistence, defined as those behaviors directed to solving the problem, such as interacting with the apparatus in different ways (e.g., Chow, 
Lea, \& Leaver, 2016; Marshall-Pescini, Rao, Virányi, \& Range, 2017). Specifically, more persistent animals spend more time focusing on the apparatus and gaze less towards people (Brubaker, Dasgupta, Bhattacharjee, Bhadra, \& Udell, 2017; Marshall-Pescini et al., 2017). In addition, dogs can adapt their gazing behavior according to variations in their environment. For instance, they are sensitive to the attentional state of the people present during the task, gazing more towards someone facing them than toward someone standing backwards (Marshall-Pescini, Colombo, Passalacqua, Merola, \& Prato-Previde, 2013).

An important interrogation regarding dogs' ability of asking for help is whether they are able to take into account prior experiences with people when choosing whom to turn to. For instance, Horn, Virányi, Miklósi, Huber, and Range (2012) confronted dogs with an apparatus that could be either empty or blocked. They had been previously exposed to two experimenters with different abilities, and each one could solve one of two problems: one filler who rebaited the apparatus when it was empty, and one helper who unblocked it. However, dogs did not take this distinction into account when selecting who to ask for help according to the type of problem they encountered (apparatus empty or apparatus blocked). It is possible that this task may have been too difficult and the animals could not understand properly the distinction between experimenters or between the actions needed to access the food (either to fill or to unlock the apparatus). However, they spent more time close to the filler when the apparatus was empty, which the authors interpreted as a rudimentary understanding of each experimenter's specific abilities. Nevertheless, it would be possible that dogs did not understand the experimenters' roles, and they expressed a preference for the one who had manipulated the food and was associated with the reward.

Similar results were obtained by Piotti, Spooner, Jim, and Kaminski (2017), when dogs observed one experimenter being skillful (i.e., successfully unblocking an apparatus and obtaining food) and one being unskilled (i.e., trying without success). When they were later confronted with an unsolvable task, however, dogs did not show a preference for either person when asking for their help. The authors then set out to investigate whether dogs would turn to a person who had been nice to them compared with someone who previously ignored them, but once again the animals showed no preference between them during the unsolvable task. They concluded that dogs appear unable to form a reputation of the people during such tasks and thus are not selective when asking for help. Like the previous study, the task was also quite complex, so it may be possible that dogs did not fully understand it or did not discriminate the differential characteristics of the experimenters. Moreover, the authors did not evaluate this discrimination.

In contrast to these findings, Kiss, Kovács, Szánthó, and Topál (2018) investigated whether dogs were able to differentiate between unfamiliar people according to their similarity to their owners. To this end, they were confronted with people who either acted as their owner (i.e., behavioral characteristics including language and motion patterns) or wore a similar piece of clothing to one worn by the owner (i.e., unfamiliar arbitrary group marker). Later, during a task in which a toy was inaccessible, they gazed more to the person whose behavioral characteristics were similar to the owner, but discrimination was weak regarding the arbitrary group marker. These results suggest that dogs could accomplish some form of social categorization in order to select potential cooperators to ask for their help, taking into account behavioral characteristics similar to their owner. Furthermore, Heberlein, Manser, and Turner (2017) found that dogs indicated the location of hidden food more often to a person who had cooperated with them compared with someone who had been competitive. This study was not an unsolvable task but rather one of showing - a kind of task in which the animal watches food being hidden while a person is outside the room. Once the person enters, the animal can indicate the location of the food with its gaze or body position. After extensive training sessions, dogs learnt the distinction between a cooperative experimenter who gave them food and a competitive one who kept it for herself. Later, during the showing task, dogs could take the experimenter to an empty place, or one containing food. In this situation, dogs were able to take into account their prior experience with people to direct their communicational attempts. Specifically, they guided the cooperative experimenter more often than the competitive one to the food. What is more, they led the competitive experimenter more often to the empty place, which was interpreted as a deceptive-like behavior.

Considering the inconsistency in previous results, and the fact that dogs may not have fully understood the complex tasks used during prior studies, we aim to evaluate whether dogs, when confronted with an unsolvable task, would selectively ask for help from a person that behaved generously with them in a previous scenario. To this end, we will use the protocol of Carballo, Freidin, Putrino, Shimabukuro, Casanave and Bentosela (2015; Carballo, Freidin, Casanave, $\&$ Bentosela, 2017), in which dogs were able to differentiate between a generous experimenter and a selfish one, and preferred to interact with the generous one after repeated short interactions with each of them. In these works (Carballo et al., 2015; Carballo et al., 2017), the generous experimenter pointed to a baited bowl and allowed the dog to eat from it, while the selfish one pointed to the baited bowl but took the food when the dog was about to eat it and ate it herself. Importantly, this is a simple discriminative task in which dogs do not need to make complex inferences about the physical aspects of the problem, as in Horn et al. (2012) or Piotti et al. (2017). Moreover, similar to Heberlein et al. (2017), there will be a preference test before the problem-solving task to ensure that dogs understood the previous part and preferred to interact with the generous person. 
The ability to assess the reputation of other individuals is fundamental for social species, given that it is helpful to recruit the best cooperative partners during cooperative exchanges (Melis, Hare, \& Tomasello, 2006). It has been proposed that dogs have acquired a set of social communicative skills during domestication that allowed them to use human social cues, such as pointing. Following this line of thought, some authors suggest that certain dogs might have been able to use subtle social cues to predict human actions and thus had a selective advantage compared with the rest of the population, which led to the evolution of these social skills (Hare, Brown, Williamson, \& Tomasello, 2002). In addition, it is commonly assumed that being able to differentiate or categorize between prosocial and antisocial individuals is a useful skill to predict the future behavior of others (Subiaul, Vonk, Okamoto-Barth, \& Barth, 2008), which in turn could help individuals avoid harmful interactions and select cooperative partners, increasing their fitness (Abdai \& Miklósi, 2016).

Given that dogs shared their ecological niche with humans and received most resources from them, it would have been particularly relevant to discriminate between generous and selfish people and selectively engage in interactions with prosocial humans, avoiding antisocial ones who may harm or ignore them (Nitzschner, Melis, Kaminski, \& Tomasello, 2012). Therefore, it would be useful for dogs to take into account previous experiences with people to select whom to ask for help during the unsolvable task. We hypothesized that, after learning to discriminate between a selfish and a generous experimenter, family dogs would selectively ask for help (i.e., turning to, gazing, alternating their gaze between the experimenter and the apparatus, approaching the experimenter) from the generous experimenter in a subsequent unsolvable task.

\section{Method}

\section{Ethical statement}

Ethical approval was obtained from the Comisión Institucional para el Cuidado y Uso de Animales de Laboratorio (CICUAL-Institutional Animal Care and Use Committee) from the Instituto de Investigaciones Médicas (Medical Research Institute; Res. No. 087-18). Owners consented to the participation of their dogs in this study.

\section{Subjects}

We assessed 57 domestic dogs, five of which could not participate due to excessive fear of the experimenters, and two of which were uninterested in the task. Additionally, as we wanted to make sure that dogs had differentiated between the experimenters before being confronted with the unsolvable task, 21 dogs did not participate in the unsolvable task because they did not select the generous experimenter during the preference test (19 choose the selfish experimenter and two did not chose). Finally, we discarded two dogs because they did not retrieve the food in the solvable trials of the unsolvable task and one dog due to a camera malfunction.

Therefore, the final sample consisted of 26 domestic dogs (mean age $=4.14$ years, $S D \pm 2.18$ years), 13 females and 13 males, from several dog breeds (three Golden Retriever, two Boxers, two Poodles, two Greyhounds, one American Staffordshire Terrier, one Bracco, one Schnauzer, one Rottweiler, and 13 mixed breeds). All dogs had lived with their family for at least a year and had no training experience or only a basic obedience level. None of them had prior experience with a similar problem-solving task. To increase their motivation during testing, we asked the owners to not feed them in the 6 hours prior to the test; water was available ad libitum.

\section{Materials}

Dogs were evaluated in a quiet room in their homes $(n=22)$ or in a canine daycare facility familiar to them $(n=4)$. There were four experimenters (Es) unfamiliar to the dogs: one generous, one selfish, one handler, and one helper during the testing (see Procedure). The owner was absent during the task.

For the discrimination of human attitudes task, the experimental setup consisted of two chairs placed $75 \mathrm{~cm}$ apart from each other, and two identical opaque bowls $(20 \mathrm{~cm}$ diameter and $8 \mathrm{~cm}$ height). One E stood between the chairs, giving the pointing signal, while the dog was kept on a leash by the handler $1.5 \mathrm{~m}$ away. To control for odor cues, each bowl had a double bottom in which five pieces of chicken were hidden. The situation was filmed with a SONY DCR-SR88 placed on a tripod behind the handler. For this phase, the reward used was cooked chicken. During the preference test, the chairs were removed.

For the problem-solving task, we used a transparent plastic container $(15 \times 15 \mathrm{~cm})$ that had a lid that could be locked for the unsolvable trial. This container was fixed to a wooden board $(61 \times 29 \mathrm{~cm})$ to prevent it from slipping. The dog was held by the handler $1 \mathrm{~m}$ away from the container. During the unsolvable trial, the Es stood on each side of the board (see Fig. 1). The reward was changed to cooked liver in order to maintain the dogs' motivation. The task was filmed using two SONY DCR-SR88 cameras placed in tripods in opposite sides of the room to film the dog from both directions.

\section{Procedure}

The protocol consisted of two tasks: (1) discrimination of human attitudes and (2) problem solving (see Table 1). 


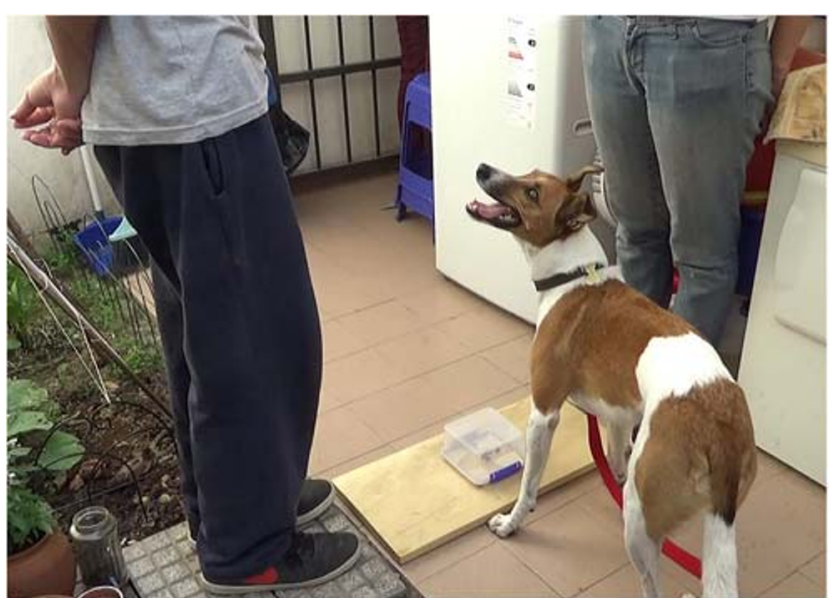

Fig. 1 Image of the experimental setting during the unsolvable task

\section{Discrimination of human attitudes}

The procedure was similar to Carballo et al. $(2015,2017)$ and comprised two phases: a discrimination task and a preference test.

Discrimination task. We used an object choice task in which dogs had to find hidden food in one of two bowls following the pointing signals of an E. Each dog received two blocks of six trials with each $\mathrm{E}$ (generous and selfish). Starting order and baited side were counterbalanced across subjects and trials, respectively. The Es included one male and one female to facilitate discrimination between them (Carballo et al., 2015). For half of the subjects, the generous attitude was played by the man, and for the other half by the woman.

- Generous E trials. The E stood between the chairs, put the bowls down (one baited) on them at the same time, and called the dog by its name using a cheerful tone. Once the dog payed attention, the E used proximal static pointing (arm and index finger extended towards the baited bowl), while leaning slightly towards the baited side and verbalizing "(Dog's name), look how yummy! Look how yummy!" If the dog picked the baited bowl, the E allowed it to eat and congratulated it verbally ("Well done!"). If the dog picked incorrectly, the E called its attention back to the baited bowl until it went to the correct one. If $15 \mathrm{~s}$ after the start of the pointing signal the dog did not approach any bowl at less than $10 \mathrm{~cm}$ (i.e., no choice), the $\mathrm{E}$ got the bowl close to the dog and let it eat while reinforcing it verbally.

- Selfish $\boldsymbol{E}$ trials. The E stood between the chairs, put the bowls down (one baited) on them at the same time, and called the dog by its name using a neutral tone. Once the dog payed attention, the E used proximal static pointing (arm and index finger extended towards the baited bowl), and remained silent. If the dog picked the baited bowl, the E took the reward, showed it to the dog, and ostensibly ate it while saying "Mmm," as if savoring the food. If the dog picked incorrectly, the E called its attention back to the baited bowl until it went to the correct one. If $15 \mathrm{~s}$ after the start of the pointing signal the dog did not approach any bowl at less than $10 \mathrm{~cm}$ (i.e., no choice), the $\mathrm{E}$ got the bowl close to the dog, showed it the food, and ate it. At the end of each trial, the handler gave a piece of food to the $\mathrm{dog}$, so the same amount of rewards was consumed on each condition.

The intervals were $20 \mathrm{~s}$ between trials and $1 \mathrm{~min}$ between blocks. After the training phase ended, the chairs were removed.

Preference test. This test took place $1 \mathrm{~min}$ after the end of the previous phase and lasted $15 \mathrm{~s}$. Both Es stood side to side, $1 \mathrm{~m}$ apart, facing the dog who was standing in the middle 1.5 $m$ away from them. The Es were looking at the dog, and each had a baited bowl in their hands at the chest level. If the dog approached first the generous $\mathrm{E}$, it received the food that was in the bowl. Only the dogs who chose the generous E continued onto the second task. After the test ended, the Es left the room.

\section{Problem-solving task}

After a 1 min interval, the second task started. It comprised two phases: solvable trials and unsolvable trials.

Solvable trials. While the handler held the dog $1 \mathrm{~m}$ away from the apparatus, an E who had not participated in the previous task (the helper) put a piece of liver in the container and left it unlocked so the dog could push it with its paw or muzzle

Table 1 Experimental design

\begin{tabular}{|c|c|c|c|c|c|c|}
\hline \multicolumn{5}{|c|}{ Discrimination of human attitudes } & \multicolumn{2}{|l|}{ Problem-solving task } \\
\hline \multicolumn{4}{|c|}{ Discrimination training } & \multirow{2}{*}{$\begin{array}{l}\text { Preference test } \\
1 \text { trial }\end{array}$} & \multirow{2}{*}{$\begin{array}{l}\text { Problem-solving task } \\
3 \text { trials }\end{array}$} & \multirow{2}{*}{$\begin{array}{l}\text { Unsolvable trial } \\
1 \text { trial }\end{array}$} \\
\hline 6 trials & 6 trials & 6 trials & 6 trials & & & \\
\hline G & $\mathrm{S}$ & G & $\mathrm{S}$ & Choice G vs. S & Solvable task & Unsolvable task \\
\hline
\end{tabular}

Note. $\mathrm{G}=$ generous; $\mathrm{S}=$ selfish. The starting order of the attitudes $(\mathrm{G}$ and $\mathrm{S}$ ) was counterbalanced across subjects as the gender of the experimenters 
and get the reward. This was repeated for three consecutive trials. Each trial had a maximum duration of 1 min or until the dog ate the food. Dogs had to retrieve the food in two out of the three trials to be able to continue onto the final phase.

Unsolvable trial. Immediately afterwards, the helper put three pieces of liver in the container and locked it so dogs were unable to retrieve the food. Then the helper left the room, while the generous and the selfish Es took their places on each side of the board. The side in which each E stood was counterbalanced across subjects. Once they were ready, the handler freed the dog from a starting point that was set equidistantly, $2 \mathrm{~m}$ away from the Es, and left the room, signaling the start of the test. The Es stood quietly, with their hands behind their backs and looking to a point in the horizon without emitting any signal. The dog was free to interact with them and the apparatus for $3 \mathrm{~min}$. Once the task ended, the helper came back and opened the container so the dogs could eat the rewards.

\section{Data analysis}

Both during the preference test and during the unsolvable task, a choice was established when the dogs first directed their head towards one E. Both measures were coded live and then confirmed through the video recordings, with an excellent agreement between Es.

To asses if dogs chose the $\mathrm{G}$ experimenter in the discrimination task above chance level ( 0.5$)$, we used a binomial test. The same analysis was used for the choice in the unsolvable task. Furthermore, to evaluate what aspects of the situation were influencing dogs' choices, we ran a binary generalized linear model (GLM) with $\log$ as a linking function with the dogs that chose one of the Es. As the dependent variable we used the side dogs chose $(0=$ left, $1=$ right $)$ and added the owner's gender (male/female), and the Es' attitude (selfish/ generous), the Es' ID (male/female), and the interaction between the two (Es' attitude/ID) as factors. We also assessed the effect size of the preference for the $G$ experimenter (Cohen's $d$ ) and ran a power analysis to compute the achieved power using the $\mathrm{G}^{*}$ Power software (Version 3.9.1).

Some results suggested an effect of owner gender on dogs' choices, so a new analysis was carried including the gender of the owner and the Es. To accomplish this, we ran a second binomial GLM with the choices as a function of the Es' ID (female $=0$; male $=1$ ) as a dependent variable and the owner's gender as a factor. We also assessed the effect size and ran a power analysis using the $G^{*}$ Power software (Version 3.9.1). Furthermore, with the dogs' choice as a function of the Es' ID, we ran a binomial test to assess whether dogs chose the male/ female $\mathrm{E}$ above chance level.

The 3 min of the unsolvable task were divided into six 30-s blocks and analyzed using frame-by-frame samples at a rate of two frames per second $(0.50 \mathrm{~s})$. During this phase, several variables were coded. A second observer measured $37.5 \%$ of the data, with a high interobserver reliability for all variables (rhos $>.98$, except for gaze alternations where rhos $>$ $.75, p \mathrm{~s}<.001)$.

- Time (s) gazing at each E (G \& S): The dog's head faced towards one E.

- Number of gaze alternations towards each $\mathrm{E}(\mathrm{G} \& \mathrm{~S})$ : The dog's head faced consecutively to one $\mathrm{E}$ and the apparatus, or vice versa.

- Time (s) in contact with each E (G \& S): Any part of the dog's body was in contact with one $\mathrm{E}$.

- Time (s) interacting with the apparatus: Behaviors aimed at obtaining the food, such as grasping, scratching, nosing, biting, and pushing.

We ran a generalized linear mixed model (GLMM) with each dependent variable: gaze, gaze alternation, and time in contact with the E. We included the owner's gender (male/female), the Es' attitude (selfish/generous), the Es' ID (male/female), and their interactions as factors. To analyze how each behavior towards the $\mathrm{E}$ changed across time, we also added block and block within Es' attitude as fixed factors. Finally, we included the subject ID as a random factor. To measure the time interacting with the apparatus, we included block as a fixed factor and subject ID as a random factor.

All tests were two tailed $(\alpha=.05)$. The data were analyzed with the statistics program SPSS (Version 20) and figures were created with GraphPad Prism (Version 7.0).

\section{Results}

\section{Choices}

For the preference test, 29 out of 50 dogs chose the G (58\%), 19 picked the $\mathrm{S}(38 \%)$, and two (4\%) did not make a choice and were discarded from the analyses. Taking in consideration only those dogs that choose one of the Es $(n=48)$, we observed that, overall, dogs showed a preference for the G over the S (binomial test: $p=.02, N=48$, power $=.91$ ). Table 2 describes dogs' choices as a function of side, Es' attitude, and Es' ID.

Table 2 Dogs' choices in the preference test as function of side, experimenters' attitude and ID

\begin{tabular}{|c|c|c|c|c|c|}
\hline \multicolumn{2}{|c|}{$\begin{array}{l}\text { Experimenter's } \\
\text { attitude }\end{array}$} & \multicolumn{2}{|c|}{ Experimenters' ID } & \multicolumn{2}{|l|}{ Side } \\
\hline Generous & Selfish & Female & Male & Right & Left \\
\hline 29 & 19 & 25 & 23 & 25 & 23 \\
\hline
\end{tabular}


To analyze what determined dogs' choices, we ran a GLM with the side (left $=0$; right $=1$ ) as the dichotomous output and the owner's gender, the Es' attitude, and the Es' ID and the interaction between Es' attitude and ID as factors. We observed no side preference, and none of the included parameters had an effect on dogs' choices-intercept: $\chi^{2}(1)=.63, p$ $=.42$; Es' attitude: $\chi^{2}(1)=1.18, p=.27$; Es' ID: $\chi^{2}(1)=.02, p$ $=.88$; Attitude $\times$ ID: $\chi^{2}(1)=.72, p=.39$; owner's gender: $\chi^{2}(1)=.32, p=.57$. We computed the effect size of the choice as a function of the Es' attitude (Cohens' $d=.28$ ), and with this we computed the power of our results with an achieved power of .27.

Regarding the choice during the unsolvable task, out of 26 dogs, 10 dogs choose $\mathrm{G}(38 \%), 15$ chose $\mathrm{S}$ (57\%), and one did not choose (4\%). Unlike the first choice, there was no preference for the $\mathrm{G}$ over the $\mathrm{S}$ (binomial test, $N=25, p=.1$, power $=.42$ ). Table 3 describes dogs' choices as a function of side, Es' attitude, and Es' ID.

The model confirms the lack of preference for the $\mathrm{G}$ experimenter, $\chi^{2}(1)=.00, p=.98$, Cohen's $d=.01$, power $=.05$. Furthermore, we did not observe a side bias, $\chi^{2}(1)=.33, p=$ .56 , a preference for either the female or the male $\mathrm{E}, \chi^{2}(1)=.1$, $p=.75$, nor an effect of interaction between the Es' attitude and ID, $\chi^{2}(1)=.78, p=.37$. Surprisingly, we found an effect of owner gender, $\chi^{2}(1)=6.44, p=.01$, as 13 of the 17 female owned dogs chose the $\mathrm{E}$ on the left side and seven of the eight male owned dogs chose the $\mathrm{E}$ on the right side. Nevertheless, this last result should be interpreted with caution, given that the final sample was unbalanced concerning the owners' gender as there were 17 female owned dogs and eight male owned dogs.

Finally, we inquired whether female owned dogs preferred the female $\mathrm{E}$ and vice versa. We further assessed the role of the owner and experimenter gender on dogs' choices running a second GLM with the dogs' choice as a function of the E's ID (male/female) as a dependent variable and owner's gender as a predictor. We did not find any significant effect of the owner gender on dogs' choices, $\chi^{2}(1)=.26, p=.60$, Cohens' $d=.21$, power $=.18$. The model also yielded a tendency towards significance that suggests that dogs chose the female over the male E, $\chi^{2}(1)=3.14, p=.07$, Cohen's $d=.17$, power $=.93$, regardless of the owners' gender. We tested this preference with a binomial test, which confirmed this result $(p=.03, N$ $=25$, power $=.93$ ).

Table 3 Dogs' choices in the preference test as function of side, experimenters' attitude, and ID

\begin{tabular}{|c|c|c|c|c|c|}
\hline \multicolumn{2}{|c|}{$\begin{array}{l}\text { Experimenter's } \\
\text { attitude }\end{array}$} & \multicolumn{2}{|c|}{ Experimenters' ID } & \multicolumn{2}{|l|}{ Side } \\
\hline Generous & Selfish & Female & Male & Right & Left \\
\hline 10 & 15 & 17 & 8 & 11 & 14 \\
\hline
\end{tabular}

\section{Gazing behavior}

Descriptive statistics for the behaviors measured are displayed in Table 4. Dogs gazed more at $\mathrm{G}$ than $\mathrm{S}, F(1,298)=5.92, p=$ .01 (see Fig. 2) and also gazed more at the female $E$ than at the male E, $F(1,298)=4.21, p=.04$ (see Fig. 2). Interestingly, we found a marginally significant interaction between Es' attitude and ID, $F(1,298)=3.76, p=.053$, as dogs gazed more at $\mathrm{G}$ when she was female. No effects of block, $F(5,298)=1.06, p$ $=.38$, or Block $\times$ Es' Attitude, $F(5,298)=.36, p=.08$, were found.

Regarding gaze alternation, we found a significant effect of block, $F(5,292)=3.74, p=.003$, but no effect of block within each Es' attitude, $F(5,292)=.38, p=.85$. Descriptively, gaze alternation decreased along trials irrespectively of the Es' attitude. No other significant differences were found (all $p \mathrm{~s}>$ $.25)$.

\section{Contact with the experimenters}

We found an effect of the E's Attitude $\times$ ID interaction, as dogs spent more time in contact with the female $\mathrm{E}$ than the male $\mathrm{E}$ when she played the $\mathrm{G}$ role and less time in contact with her when she played the $\mathrm{S}$ role, $F(1,297)=5.29, p=.02$. No other significant differences were found (all $p \mathrm{~s}>.05$ ).

\section{Interaction with the apparatus}

Time dogs spent interacting with the apparatus decreased along blocks of trials, $F(5,150)=19.46, p<.001$ (see Fig. 3).

\section{Discussion}

The aim of this study was to assess whether dogs would take into account their prior experience with a generous and a selfish $\mathrm{E}$ in order to selectively ask for help during a problemsolving task. To this end, dogs first underwent a discrimination task in which they learnt to differentiate between a generous and a selfish E. After that, there was a preference test aimed to confirm that they had indeed discriminated between the Es. Only those dogs who first chose the generous E continued to the problem-solving part of the study. During this task, the dogs were confronted with a locked container while they were free to interact with the apparatus and the Es. Results indicate that, during the problem-solving situation, dogs did not first select the generous E, although they spent more time gazing at them that at the selfish one.

Dogs were able to significantly choose the $\mathrm{G}$ partner during the preference test that came immediately after training, as they did in prior studies (Carballo et al., 2015; Carballo et al., 2017). This finding suggests that they properly learnt about the different attitudes of the Es and could discriminate 
Table 4 Mean (in bold) and standard deviations (s) of the behaviors assessed

\begin{tabular}{llllllll}
\hline Measure/block & B 1 & B 2 & B 3 & B 4 & B 5 & B 6 & Total \\
\hline Gaze G & $\mathbf{. 7 8} \pm 1.74$ & $\mathbf{2 . 4 0} \pm 4.84$ & $\mathbf{1 . 8 0} \pm 4.08$ & $\mathbf{2 . 3 8} \pm 3.91$ & $\mathbf{2 . 5 5} \pm 6.00$ & $\mathbf{2 . 0 0} \pm 5.35$ & $\mathbf{1 1 . 9 4} \pm 20.58$ \\
Gaze S & $\mathbf{. 6 5} \pm 1.07$ & $\mathbf{1 . 4 4} \pm 2.34$ & $\mathbf{1 . 4 4} \pm 2.95$ & $\mathbf{1 . 5 0} \pm 3.11$ & $\mathbf{. 9 2} \pm 1.83$ & $\mathbf{1 . 0 9} \pm 3.58$ & $\mathbf{7 . 0 5} \pm 9.77$ \\
Alternation G & $\mathbf{. 2 0} \pm .40$ & $\mathbf{. 1 2} \pm .33$ & $\mathbf{. 0 2} \pm .10$ & $\mathbf{. 1 0} \pm .25$ & $\mathbf{. 0 6} \pm .16$ & $\mathbf{. 0 2} \pm .10$ & $\mathbf{. 5 0} \pm .77$ \\
Alternation S & $\mathbf{. 1 5} \pm .30$ & $\mathbf{. 0 3} \pm .13$ & $\mathbf{. 0 1} \pm .09$ & $\mathbf{. 0 7} \pm .23$ & $\mathbf{. 0 5} \pm .21$ & $\mathbf{. 0 3} \pm .13$ & $\mathbf{. 3 8} \pm .62$ \\
Contact G & $\mathbf{. 3 2} \pm 1.04$ & $\mathbf{. 1 0} \pm .35$ & $\mathbf{. 1 7} \pm .56$ & $\mathbf{. 2 3} \pm .91$ & $\mathbf{. 8 6} \pm 2.56$ & $.94 \pm 2.86$ & $\mathbf{2 . 6 3} \pm 5.50$ \\
Contact S & $\mathbf{. 1 5} \pm .46$ & $\mathbf{. 3 4} \pm 1.57$ & $\mathbf{. 6 3} \pm 1.93$ & $\mathbf{. 0 1} \pm .09$ & $\mathbf{. 3 6} \pm 1.48$ & $\mathbf{. 0 3} \pm .19$ & $\mathbf{1 . 5 5} \pm 3.53$ \\
Interaction apparatus & $\mathbf{1 8 . 1 3} \pm 9.35$ & $\mathbf{1 1 . 9 4} \pm 11.81$ & $\mathbf{4 . 8 6} \pm 8.14$ & $\mathbf{3 . 4 6} \pm 5.76$ & $\mathbf{3 . 9 0} \pm 6.64$ & $1.96 \pm 5.00$ & $\mathbf{4 4 . 2 6} \pm 27.85$ \\
\hline
\end{tabular}

Note. $\mathrm{B}=$ block; $\mathrm{G}=$ generous; $\mathrm{S}=$ selfish

between them. Therefore, the fact that they did not turn first to the $\mathrm{G}$ one during the unsolvable task may not be explained by a lack of understanding of the different roles of the human partners.

Dogs gazed significantly more towards the generous E during the task. This suggests they did develop a preference for the generous $\mathrm{E}$, although this was not conveyed in their first choice or in the time interacting with the E. This result proposes that dogs are able to take into account people's attitudes and react differently according to them. This would have a great adaptative value, as humans provide relevant resources for them such as food, water, and shelter (e.g., Udell, Dorey, \& Wynne, 2010). Therefore, being able to select those partners that are more likely to provide access to such resources would be a valuable skill for dogs.

On the other hand, dogs tended to prefer the female E over the male one and spent significantly more time gazing at her during the task. Several studies suggest that dogs easily differentiate between men and women and react differently towards them. For instance, they are quicker to approach a woman who is sitting down passively (Lore \& Eisenberg, 1986) and show stronger emotional reactions (i.e., tail wagging, pawing, ears back) to men than to women (Wickens et al., 1995). Moreover, Wells and Hepper (1999) indicate that the time dogs spent gazing and barking towards an experimenter tended to decrease faster when she was female. In addition, Hennessy, Davis, Williams, Mellott, and Douglas (1997) found that brief interactions with women, but not with men, decreased dogs' cortisol levels during their first days in a shelter. All in all, these findings may help to explain why dogs preferred to turn to a woman and spent more time gazing at her during the unsolvable task. Probably, in their everyday life, dogs have more positive interactions with women or find the same interactions more positive when they come from women, as suggested by Hennessy et al. (1997).

Moreover, some results suggest an interaction between the Es' ID (male/female) and their attitude (generous/selfish). In particular, dogs gazed more and spent more time in contact with the female $\mathrm{E}$ when she was generous. This suggests a synergic effect of these factors on dogs' behavior. Therefore, dogs may take into account both the consequences of immediate brief encounters and lifelong reinforcing experiences when deciding whom to ask for help. In line with this, as most dogs were owned by females, it is possible that they generalized
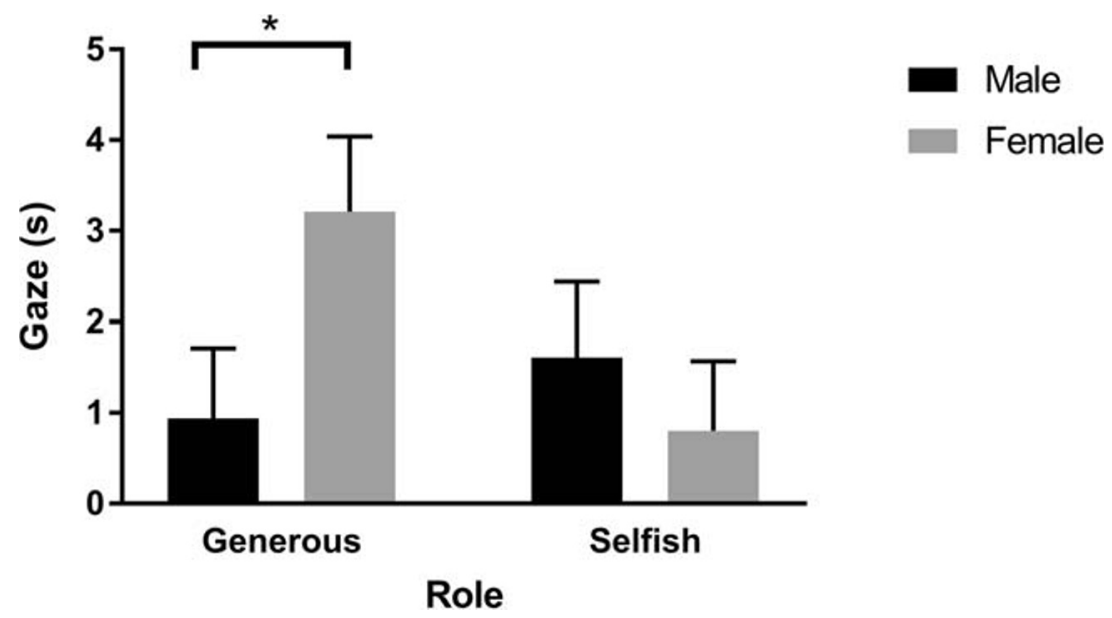

Fig. 2 Mean and standard error of the time in seconds that dogs spent gazing at the experimenter during the unsolvable tasks as a function of the experimenter's ID and attitude 


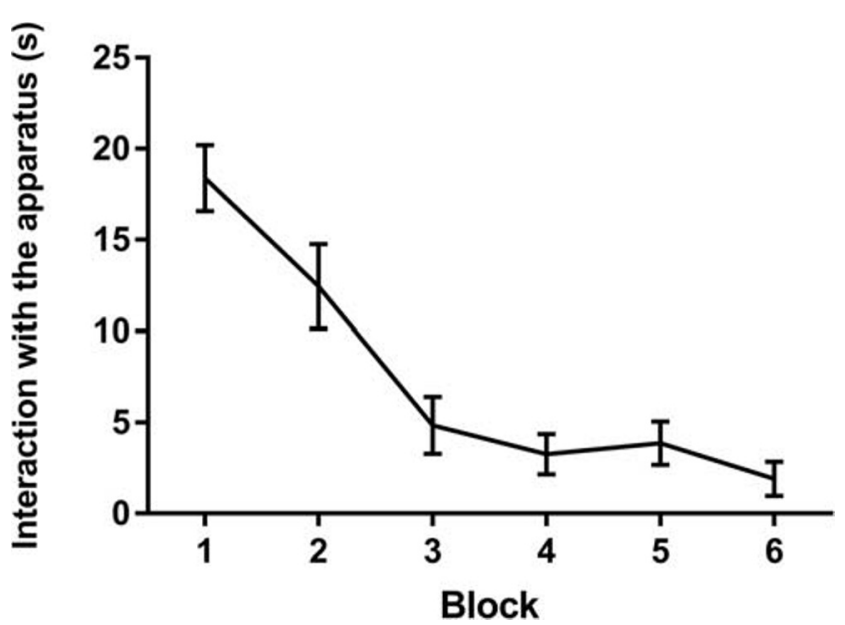

Fig. 3 Mean and standard error of the time in seconds that dogs spent interacting with the apparatus along blocks during the unsolvable task

their learnt responses with their owner to the female E. This interpretation is in accordance with the results from Kiss et al. (2018), which showed that dogs preferred to interact with those experimenters who shared some behavioral attitudes with their owners. Nevertheless, this must be interpreted with caution, as we did not find a direct effect of the owner's gender on dogs' choices. Furthermore, the Es may differ in other aspects beside gender, so this hypothesis should be tested explicitly.

Surprisingly, no significant differences were found in gaze alternation towards the Es, due to neither their attitude nor gender. This may be due to the relatively low appearance of this behavior during the task.

The present results are in accordance with those obtained in a showing task (Heberlein et al., 2017), where dogs were subjected to extensive training in order to discriminate people's attitudes. Therefore, the amount of training dogs receive during the task seems to be key for this ability to manifest. However, in line with previous studies (Horn et al., 2012; Piotti et al., 2017), the dogs did not take the Es' attitudes into account when first selecting helpers during the unsolvable task, as there was no preference for the generous E.

Finally, a significant effect of block was found in the time spent interacting with the apparatus, as dogs interacted less with it as trial duration increased. This is expected, as their attempts to open the container were not successful because it was locked, which led to the extinction of this behavior as time went by (Skinner, 1953). Gaze alternation also decreased across trials, which could be similarly attributed to a process of extinction across the task.

All in all, dogs may not take into account prior attitudes of a partner to first select a helper. However, they both gazed and were in contact more with the generous $\mathrm{E}$ during the task, suggesting a certain degree of preference for this person. The ability to quickly build a reputation about people to subsequently guide their decisions in a problematic situation, could be an important feature of dogs' sociocognitive skills and could have been of great relevance for their incorporation to human societies.

Acknowledgements We would like to thank to the owners who kindly allowed their dogs to participate in this study. This research was supported by CONICET (Grant 2013 No. 11220130100182) and AGENCIA NACIONAL DE PROMOCIÓN CIENTÍFICA Y TECNOLÓGICA (Grant PICT 2014, No. 0883). The authors declare no conflict of interests.

\section{References}

Abdai, J., \& Miklósi, Á. (2016). The origin of social evaluation, social eavesdropping, reputation formation, image scoring or what you will. Frontiers in Psychology, 7, 1772.

Brubaker, L., Dasgupta, S., Bhattacharjee, D., Bhadra, A., \& Udell, M. A. (2017). Differences in problem-solving between canid populations: Do domestication and lifetime experience affect persistence? Animal Cognition, 20(4), 717-723.

Carballo, F., Freidin, E., Casanave, E. B., \& Bentosela, M. (2017). Dogs' recognition of human selfish and generous attitudes requires little but critical experience with people. PLOS ONE, 12(10), e0185696. doi:https://doi.org/10.1371/journal.pone.0185696

Carballo, F., Freidin, E., Putrino, N., Shimabukuro, C., Casanave, E., \& Bentosela, M. (2015). Dog's discrimination of human selfish and generous attitudes: The role of individual recognition, experience, and experimenters' gender. PLOS ONE, 10(2), e0116314.2017. doi: https://doi.org/10.1371/journal.pone.0116314

Chow, P. K. Y., Lea, S. E., \& Leaver, L. A. (2016). How practice makes perfect: The role of persistence, flexibility and learning in problemsolving efficiency. Animal Behaviour, 112, 273-283.

Hare, B., Brown, M., Williamson, C., \& Tomasello, M. (2002). The domestication of social cognition in dogs. Science, 298(5598), 1634-1636.

Heberlein, M. T., Manser, M. B., \& Turner, D. C. (2017). Deceptive-like behaviour in dogs (Canis familiaris). Animal Cognition, 20(3), 511-520.

Hennessy, M. B., Davis, H. N., Williams, M. T., Mellott, C., \& Douglas, C. W. (1997). Plasma cortisol levels of dogs at a county animal shelter. Physiology \& Behavior, 62(3), 485-490.

Horn, L., Virányi, Z., Miklósi, Á., Huber, L., \& Range, F. (2012). Domestic dogs (Canis familiaris) flexibly adjust their humandirected behavior to the actions of their human partners in a problem situation. Animal Cognition, 15(1), 57-71.

Kiss, O., Kovács, K., Szánthó, F., \& Topál, J. (2018). Similarity between an unfamiliar human and the owner affects dogs' preference for human partner when responding to an unsolvable problem. Learning \& Behavior, 46(4), 430-441.

Leavens, D. A., Hopkins, W. D., \& Thomas, R. K. (2004). Referential communication by chimpanzees (Pan troglodytes). Journal of Comparative Psychology, 118(1), 48.

Lore, R. K., \& Eisenberg, F. B. (1986). Avoidance reactions of domestic dogs to unfamiliar male and female humans in a kennel setting. Applied Animal Behaviour Science, 15, 262-266.

Marshall-Pescini, S., Colombo, E., Passalacqua, C., Merola, I., \& PratoPrevide, E. (2013). Gaze alternation in dogs and toddlers in an unsolvable task: Evidence of an audience effect. Animal Cognition, 16(6), 933-943.

Marshall-Pescini, S., Passalacqua, C., Barnard, S., Valsecchi, P., \& PratoPrevide, E. (2009). Agility and search and rescue training differently affects pet dogs' behaviour in socio-cognitive tasks. Behavioural Processes, 81(3), 416-422.

Marshall-Pescini, S., Rao, A., Virányi, Z., \& Range, F. (2017). The role of domestication and experience in 'looking back' towards humans in 
an unsolvable task. Scientific Reports, 7, 46636. doi:https://doi.org/ 10.1038/srep46636

Melis, A. P., Hare, B., \& Tomasello, M. (2006). Chimpanzees recruit the best collaborators. Science, 311(5765), 1297-130.

Miklósi, Á., Kubinyi, E., Topál, J., Gácsi, M., Virányi, Z., \& Csányi, V. (2003). A simple reason for a big difference: Wolves do not look back at humans, but dogs do. Current Biology, 13(9), 763-766.

Miklósi, Á., Polgárdi, R., Topál, J., \& Csányi, V. (2000) Intentional behaviour in dog-human communication: An experimental analysis of showing behaviour in the dog. Animal Cognition, 3, 159-166.

Nitzschner, M., Melis, A. P., Kaminski, J., \& Tomasello, M. (2012). Dogs (Canis familiaris) evaluate humans on the basis of direct experiences only. PLOS ONE, 7(10), e46880.

Piotti, P., Spooner, R. M., Jim, H. L., \& Kaminski, J. (2017). Who to ask for help? Do dogs form an opinion on humans based on skilfulness? Applied Animal Behaviour Science, 195, 93-102.

Prato-Previde, E., \& Marshall-Pescini, S. (2014). Social looking in the domestic dog. In A. Horowitz (Ed.), Domestic dog cognition and behavior: The scientific study of Canis familiaris (pp. 101-131). Berlin, Germany: Springer. doi:https://doi.org/10.1007/978-3-64253994-7 5

Skinner, B. F. (1953). Science and human behavior. New York, NY: Simon \& Schuster.
Subiaul, F., Vonk, J., Okamoto-Barth, S., \& Barth, J. (2008). Do chimpanzees learn reputation by observation? Evidence from direct and indirect experience with generous and selfish strangers. Animal Cognition, 11(4), 611-623.

Udell, M. A. (2015). When dogs look back: Inhibition of independent problem-solving behaviour in domestic dogs (Canis lupus familiaris) compared with wolves (Canis lupus). Biology Letters, 11(9), 20150489. doi:https://doi.org/10.1098/rsbl.2015.0489

Udell, M. A., Dorey, N. R., \& Wynne, C. D. (2010). What did domestication do to dogs? A new account of dogs' sensitivity to human actions. Biological Reviews, 85(2), 327-345.

Wells, D. L., \& Hepper, P. G. (1999). Male and female dogs respond differently to men and women. Applied Animal Behaviour Science, 61, 341-349.

Wickens, S. M., Astell-Billings, I., McPherson, J. A., Gibb, R., Bradshaw, J. W. S., \& McBride, E. A. (1995). The behavioural assessment of dogs in animal shelters: Inter-observer reliability and data redundancy. In B. Potters (Ed.), Proceedings of the 29th International Congress of the International Society for Applied Ethology (pp. 127-128). Exeter, England: UFAW.

Publisher's note Springer Nature remains neutral with regard to jurisdictional claims in published maps and institutional affiliations. 